\section{Fine Structure of Apple Leaves Treated with the Sterol-inhibiting Fungicide Bitertanol}

\author{
S.V. Overton', L.D. Moore', and O.K. Miller ${ }^{2}$ \\ Virginia Polytechnic Institute and State University, Blacksburg, \\ VA 24061
}

Additional index words. Malus domestics, chloroplast, thylakoid

\begin{abstract}
Ultrastructural observations were made of leaves of apple (Malus domestics Borkh. cv. Red Delicious) 12, 24, and 72 hours following a single foliar application of the sterol-inhibiting fungicide bitertanol. Thylakoids of chloroplasts from treated leaves were swollen and irregular and chloroplasts had lost their integrity within 12 hours after treatment. Occasionally, mitochondria looked washed out, although no other changes in membrane or organelle structures were observed. Within 24 to 72 hours, moreover, thylakoids of chloroplasts from treated leaves returned to a state similar to that of the controls. However, the numbers of starch granules in the chloroplasts of treated leaves appeared to increase throughout the 72 hours and remained somewhat higher than levels in controls. Thus, bitertanol does not appear to have a lasting effect on apple leaves. Chemical name used: $\beta$-([1,1'-biplenyl]-4-yloxy)- $\alpha$-(1,1-dimethylethyl)-1H-1,2,4-trizole-1-ethanol (bitertanol).
\end{abstract}

The inhibition of sterol biosynthesis by agricultural chemicals has been 'studied extensively in animal tissues (Schroepfer, 1981) and in microorganisms (Leroux, 1982; Oelschlager et al., 1980). Little research has been-conducted with higher plants, although many of the sterol-inhibiting compounds used in agriculture reduce the growth of plants (Buchenauer and Grossman, 1977; Buchenauer and Rohner, 1981; Shive and Sisler, 1976). Moreover, triarimol, a sterol-inhibiting fungicide (SIF), and ancymidol, a plant growth retardant, are reported to interfere with ergosterol biosynthesis in fungi (Ragsdale, 1975). These compounds reportedly retarded the growth of Phaseolus vulgaris L.cv. Contender seedlings but had no noticeable effect on the qualitative and quantitative distribution of the main sterols present (Shive and Sisler, 1976). The SIFs nuarimol, triadimenol, triadimefon, and imazalil inhibited the incorporation of ${ }^{14} \mathrm{C}$ acetate into lipids of barley plants. The effects of these SIFs on the sterol fractions were analogous to those reported to occur in fungal systems (Buchenauer and Rohner, 1981), although much

Received for publication 10 July 1989. This study was supported, in part, by the Mobay Chemical Corp. The cost of publishing this paper was defrayed in part by the payment of page charges. Under postal regulations, this paper therefore must be hereby marked advertisement solely to indicate this fact.

'Dept. of Plant Pathology, Physiology and Weed Science.

${ }^{2}$ Dept. of Biology. higher-concentrations were required to achieve comparable results.

Even though SIFs are often applied throughout a growing season on numerous

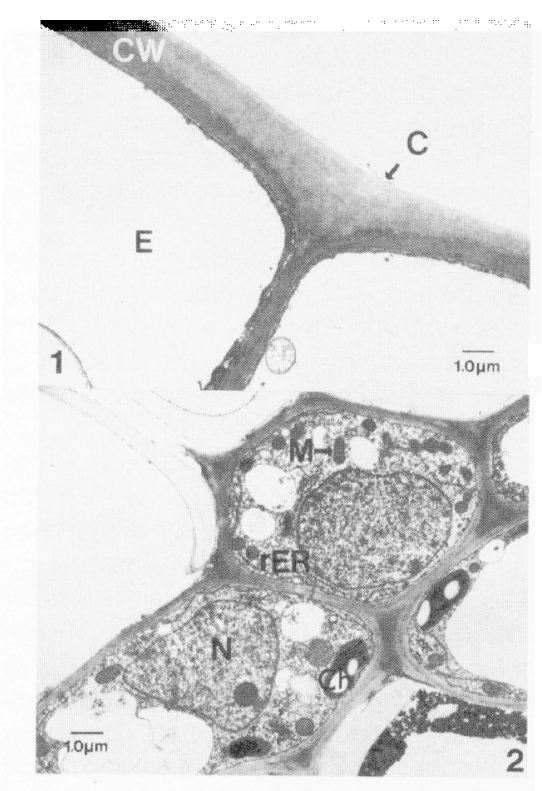

Figs. 1 and 2. Untreated 'Red Delicious' leaves. (1) Transmission electron micrograph of an untreated leaf showing a uniform cuticular layer with underlying epidermal cells; cuticle (C), cell wall $(\mathrm{CW})$, epidermis $(\mathrm{E}), \times 5600$. (2) Parenchyma cells showing internal arrangement of organelles; chloroplast (Ch), mitochondrion (M), nucleus $(\mathrm{N})$, rough endoplasmic reticulum (rER), $\times 5600$. field crops, ornamentals, and fruit crops, no studies have been conducted concerning the effects of these compounds on the ultrastructure of leaves of higher plants. The purpose of this study has been to examine the effects of the SIF bitertanol (Bayer) on the fine structure of leaves of the cultivar Red Delicious.

Four 10- to 12-year-old 'Red Delicious' apple trees were used in this study. The trees, which were selected for uniformity of size and fruit set, had not previously been treated with SIF. Twelve groups of shoots per aspect located on the north, east, west, and south aspects of each tree were used. An equal number of shoots was treated with bitertanol and maintained as controls. The bitertanol was applied with a hand sprayer to abaxial and adaxial leaf surfaces at a rate equivalent to $1.134 \mathrm{~g} \cdot \mathrm{ha}^{-1}$ The six youngest fully expanded leaves of four sprayed and unsprayed branches located at each aspect of each tree were used. Thus, there were 24 leaf samples at each aspect on each of four trees.

Four 1- to 3-mm rectangular strips intermediate between a primary vein and margin of leaves, untreated and treated with the fungicide, were removed with a razor blade 12 , 24 , and $72 \mathrm{hr}$ after fungicide application. These strips were then processed for electron microscopy by being prefixed in $4 \%$ glutaraldehyde in $0.1 \mathrm{M}$ cacodylate buffer for $5 \mathrm{hr}$

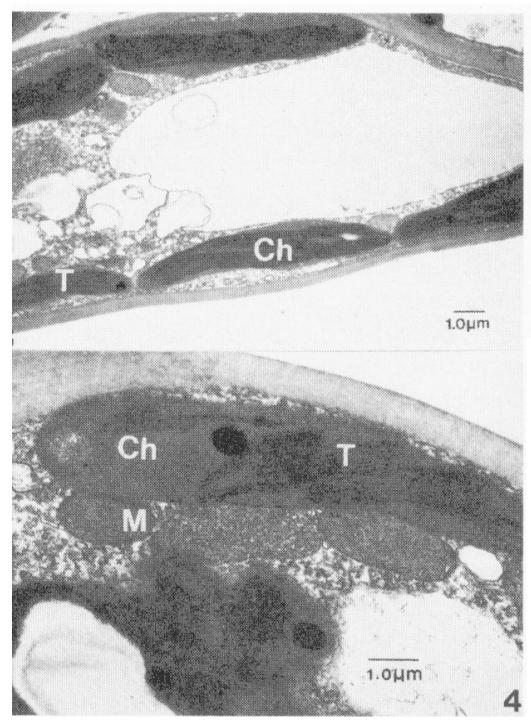

Figs. 3 and 4. Untreated 'Red Delicious' leaves. (3) Transmission electron micrograph of pali. sade parenchyma cells showing peripheral elongated chloroplasts; chloroplast (Ch), thylakoid (T), x 5200. (4) Palisade parenchyma cells showing numerous chloroplasts containing minute electron dense lipid bodies. Note spherical to elongated mitochondria; mitochondrion (M), chloroplast $(\mathrm{Ch})$, thylakoids $(\mathrm{T}), \times 8450$. 


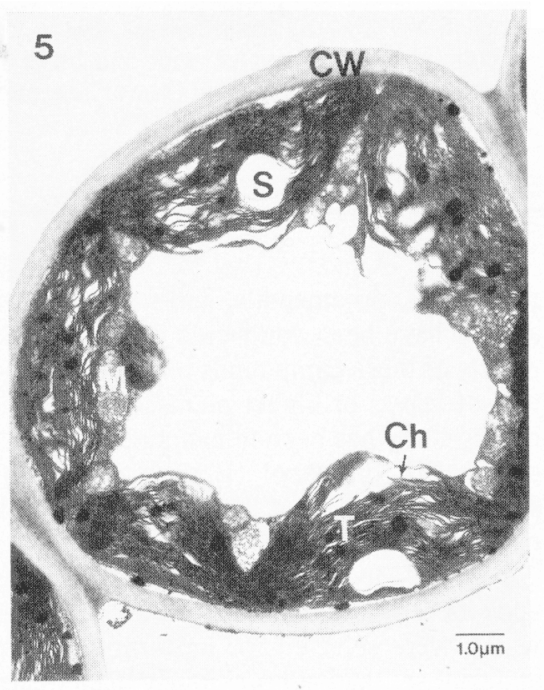

Fig. 5. 'Red Delicious' leaves $12 \mathrm{hr}$ after application of bitertanol. Palisade parenchyma cell exhibiting membrane disruption and swelling of the thylakoids. Note the increase in the number of starch granules; chloroplast $(\mathrm{Ch})$, cell wall (CW), mitochondrion (M), starch (S), thylakoids $(\mathrm{T}), \times 6600$.

at $0 \mathrm{C}$, washed in three changes of $0.1 \mathrm{M}$ sodium cacodylate buffer for $1 \mathrm{hr}$, and postfixed in sodium cacodylate buffered osmium tetroxide for $16 \mathrm{hr}$ at $0 \mathrm{C}$. The strips were then washed in three changes of $0.1 \mathrm{M}$ sodium cacodylate buffer for 45 rein, dehydrated in a graded ethanol series (30 min each) followed by an additional change of absolute alcohol for 30 rein, and then by acetone to remove the water that might be present (two changes of 10 min each). Next, the strips were stepwise infiltrated overnight and embedded in Spurr's low viscosity resin (Spurr, 1969). Blocks were cured at 70C for $24 \mathrm{hr}$. Sections were cut on a Sorvall MT2B ultramicrotome with a DuPont diamond knife, collected on 200-mesh copper grids, and double-stained with $2 \%$ uranyl acetate for 30 min and Reynold's lead citrate (Reynold, 1963) for $8 \mathrm{~min}$.

Untreated leaves possessed a uniform cuticular layer that covered the outer surface of the epidermis (Fig. 1). Chloroplasts were found primarily in the palisade and spongy mesophyll cells of the leaf (Figs. 2-4). Palisade parenchyma consisted of long columnar cells (Figs. 3 and 4). Located around the periphery of these cells were numerous spherical to elongate chloroplasts that measured $\approx 2 \times 6 \mu \mathrm{m}$. The internal structure of these chloroplasts was characterized by a system of membranes, the basic subunit of which was a thylakoid (Figs. 3 and 4). Tightly packed stacks of thylakoids, parallel to each other, were present-throughout the stroma of the chloroplasts. In addition, lipid and starch granules were located within the chloroplasts.

Twelve hours after treatment, the thylakoids of chloroplasts were swollen and irregular (Figs. 5 and 6), which gave the chloroplasts a somewhat distorted appearance. Occasionally, mitochondria were washed out (Fig. 7), while nuclei were less
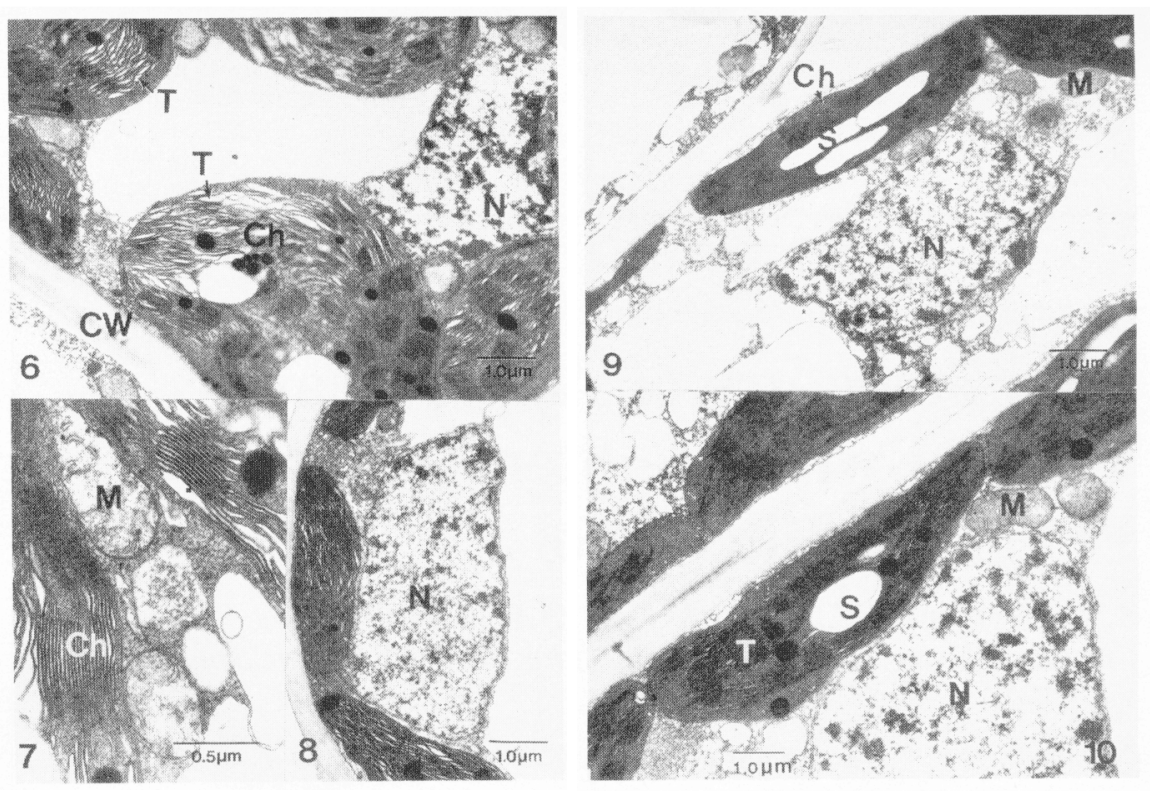

Figs. 6-8. 'Red Delicious' leaves $12 \mathrm{hr}$ after application of bitertanol. (6) Higher magnification than in Fig. 5 of chloroplasts showing membrane disruption and thylakoid swelling (arrows); chloroplast $(\mathrm{Ch})$, cell wall $(\mathrm{CW})$, nucleus $(\mathrm{N})$, thylakoids $(\mathrm{T}), \times 13,300$. (7) Mitochondria are washed-out; chloroplast $(\mathrm{Ch})$, mitochondrion $(\mathrm{M}) \times 30,800$. (8) Nucleus is less densely stained; nucleus $(\mathrm{N}), \times 9800$.

densely stained (Figs. 6 and 8).

After 24 (Fig. 9) and 72 hr (Fig. 10) the thylakoids were tightly packed and no longer swollen. Mitochondria were normal although nuclei remained somewhat washed out (Figs. 9 and 10). There appeared to be, moreover, an increase in the size and number of starch granules 12,24 , and $72 \mathrm{hr}$ following treatment with the fungicide (Figs. 5, 9, and 10). This observation was based on the examination of $\approx 50$ samples selected at random. However, a morphometrical analysis would be required to statistically substantiate this observation.

treatment of 'Red Delicious' leaves with bitertanol may lead to a temporary alteration in photosynthetic efficiency immediately after application as evidenced by the presence of swollen and irregular thylakoid membranes and loss in chloroplast integrity $12 \mathrm{hr}$ following treatment. Ashton et al. (1963) observed the disorganization of chloroplasts and cellular membranes induced by the triazine herbicide atrazine, an electron transport inhibitor. The structure of atrazine is similar to that of bitertanol in that both have N-containing rings. That bitertanol inhibited photosynthesis was not established in this study and awaits further investigation. However, the chloroplasts appeared to have recovered from the effect of the fungicide and returned to a normal state 24 and $72 \mathrm{hr}$ after treatment. It was previously observed by Overton et al. (1988) that the stigmasterol level of bitertanol-treated 'Red Delicious' leaves had decreased significantly $24 \mathrm{hr}$ after treatment. Moreover, treatments with bitertanol affected the free sterol content of apple leaves only within the initial $24 \mathrm{hr}$ after treatment
Figs. 9 and 10. 'Red Delicious' leaves 24 and $72 \mathrm{hr}$ after application of bitertanol. (9) Transmission electron micrograph of a palisade parenchyma cell showing internal organelle arrangement $24 \mathrm{hr}$ after leaf was treated. Chloroplasts and mitochondria are normal, although the nucleus is less dense as compared to those of the controls. Note the increased numbers of starch granules within the chloroplast; chloroplast (Ch), mitochondrion (M), nucleus $(\mathrm{N})$, starch (S), × 9800. (10) Palisade parenchyma cells $72 \mathrm{hr}$ after treatment showing internal arrangement of organelles. Chloroplasts and mitochondria are normal, although the nucleus remains washed-out; mitochondrion (M), nucleus $(\mathrm{N})$, starch $(\mathrm{S})$, thylakoids $(\mathrm{T}), \times 9100$.

(Overton et al., 1988). This correlated well with the observation that the thylakoids and the chloroplasts regained their integrity in the same time period.

It should be noted that in treated leaves, the nuclei were somewhat less densely stained throughout the treatment period. A more extensive time course investigation would be required to determine if such an effect is permanent and if it is detrimental. Overall, it appears that bitertanol did not have a longlasting effect on the plant sterol concentration or on the structural integrity of apple leaves. However, one must be aware that SIFs such as bitertanol might have an effect on plant metabolism if used continually for several years.

\section{Literature Cited}

Ashton, F.M., E.M. Gifford, and T. Bisalputra. 1963. Structural changes in Phaseolus vulgaris induced by atrazine. 11. Effect on fine structure of chloroplasts. Bot. Gaz. 124:336-343.

Buchenauer, H. and F. Grossman. 1977. Triadimefon: mode of action in plants and fungi. Neth. J. Plant Pathol. 83:93-103.

Buchenauer, H. and E. Rohner. 1981. Effect of triadimefon and triadimenol on growth of various plant species as well as on gibberellin content and sterol metabolism in shoots of barley seedlings. Pesticide Biochem. Physiol. 15:5870. 
Leroux, P. 1982. Phenomes de resistance de Botrytis cinerea aux fungicides. La Defense des Vegetaux 36:3-18.

Oelschlager, A.C., H.D. Pierce, A.M. Pierce, R.H

Angus, E. Quantin-Martenot, A.M. Unrau, and R. Srinwasan. 1980. The use of mutants and azasterols in studies of yeast sterol biosynthesis, p. 395-403. In: P. Benveniste, C. Costes, R. Deuce, and P. Mazliak (eds.). Biogenesis arid function of plant lipids. Elsevier/North-Holland Biomedical Press, Amsterdam.
Overton, S.V., L.D. Moore, D.M. Oreutt, K.S. Yoder, and S.A. Meredith. 1988. Influence of sterol-inhibiting fungicides on the free sterol and free fatty acid composition of Red Delicious and Jonathan apple cultivars. J. Hort. Sci. 63:183-191.

Ragsdale, N.N. 1975. Specific effects of triarimol on sterol biosynthesis in Ustilago maydis. Biochim. Biophys. Acts. 380:81-96.

Reynolds, E.S. 1963. The use of lead citrate at high $\mathrm{pH}$ as an electron opaque stain in electron microscopy. J. Cell Biol. 17:208-212.

Schroepfer, G.J. 1981. Sterol biosynthesis. Annu. Rev. Biochem. 50:585-621.

Shive, J.B. and H.D. Sisler. 1976. Effect of ancymidol (a growth retardant) and triarimol (a fungicide) on the growth, sterols, and gibberellin of Phaseolus vulgaris. Plant Physiol. 57:640-644.

Spurr, A.R. 1969. A low viscosity epoxy resin embedding medium for electron microscopy. J. Ultrastruct. Res. 26:31. 